\title{
Analgesic Effect of Harpagophytum procumbens on Postoperative and Neuropathic Pain in Rats
}

\author{
Dong Wook Lim ${ }^{\dagger}$, Jae Goo Kim ${ }^{\dagger}$, Daeseok Han and Yun Tai Kim * \\ Functionality Evaluation Research Group, Korea Food Research Institute, Seongnam 463-746, Korea \\ $\dagger$ These authors contributed equally in this work. \\ * Author to whom correspondence should be addressed; E-Mail: ytkim@kfri.re.kr; \\ Tel.: +82-31-780-9295; Fax: +82-31-709-9876.
}

Received: 12 December 2013; in revised form: 10 January 2014 / Accepted: 13 January 2014 / Published: 16 January 2014

\begin{abstract}
Harpagophytum procumbens, also known as Devil's Claw, has historically been used to treat a wide range of conditions, including pain and arthritis. The study was designed to investigate whether $H$. procumbens extracts exhibit analgesic effects in plantar incision and spared nerve injury (SNI) rats. The whole procedure was performed on male SD rats. To evaluate pain-related behavior, we performed the mechanical withdrawal threshold (MWT) test measured by von Frey filaments. Pain-related behavior was also determined through analysis of ultrasonic vocalization (USVs). The results of experiments showed MWT values of the group that was treated with $300 \mathrm{mg} / \mathrm{kg} \mathrm{H}$. procumbens extract increased significantly; on the contrary, the number of $22-27 \mathrm{kHz}$ USVs of the treated group was reduced at $6 \mathrm{~h}$ and $24 \mathrm{~h}$ after plantar incision operation. After 21 days of continuous treatment with $H$. procumbens extracts at $300 \mathrm{mg} / \mathrm{kg}$, the treated group showed significantly alleviated SNI-induced hypersensitivity responses by MWT, compared with the control group. These results suggest that $H$. procumbens extracts have potential analgesic effects in the case of acute postoperative pain and chronic neuropathic pain in rats.
\end{abstract}

Keywords: Harpagophytum procumbens; pain; hyperalgesia; allodynia; ultrasonic vocalization 


\section{Introduction}

Pain is a common symptom but very distressing feature of many diseases. In general, analgesics relieve pain by acting on the central nervous system or peripheral pain mechanisms, without significantly altering consciousness [1]. Patients suffering from this kind of pain include those with so-called nociceptive and neuropathic pain, allodynia, and hyperalgesia. [2,3]. However, pain management remains a major clinical challenge, because there is not an appropriate understanding of the mechanisms causing and maintaining pain and effective treatments [4].

Therapeutic drugs for treating pain have limited effectiveness and safety [5]. Repetitive use of non-steroidal anti-inflammatory drugs (NSAIDs) may cause adverse effects such as gastrointestinal lesions or renal and liver failure [6]. Furthermore, current analgesic drugs, even including the opioids, cannot ease the pain easier of painful conditions like neuropathic pain [7]. Therefore, it is necessary to search for new, effective and safe analgesics among natural products derived from secondary metabolites. Natural products are considered an incomparable source of molecular diversity that has led to the discovery of drugs in modern medicine, especially for pain treatment [8-10].

Harpagophytum procumbens, also known as Devil's Claw, is a herbaceous plant species that has the high level of medicinal use value in the Kalahari Desert region of southern Africa [11]. It has historically been used to treat several symptoms such as fever, malaria, indigestion and pain [12]. In addition, it was demonstrated that $H$. procumbens extracts have beneficial effects in the case of rheumatic diseases according to animal and clinical studies [13]. There have been reports verifying the anti-inflammatory effects of $H$. procumbens extracts on acute or sub-chronic inflammation in a rat model [14,15]. However, no studies have been made of the effect of $H$. procumbens extracts on surgical incision postoperative pain or neuropathic pain in in vivo models. Therefore, the present study was designed to investigate whether $H$. procumbens extracts exhibit anti-nociceptive effects in the postoperative pain through plantar incision model [16] and on the spared nerve injury (SNI) rat model of neuropathic pain [17]. To evaluate pain-related behavior, we performed the mechanical withdrawal threshold (MWT) test measured by von Frey filaments, and pain-induced ultrasonic vocalizations (USVs) have been examined by ultrasonic microphones [18]. Further, the results have been compared with those of naproxen, a NSAID [19].

\section{Results and Discussion}

\subsection{Effects of H. Procumbens Extracts on Mechanical Hyperalgesia Induced by Plantar Incision}

The analgesic activity of $H$. procumbens extracts was determined using the postoperative pain model in rats. Postoperative pain in humans can be mimicked by plantar incision in rats [16]. Incision of the plantar surface of the hind paw produced a significant reduction in the mechanical withdrawal threshold (MWT), as measured using the von Frey assay, and non-steroidal anti-inflammatory drugs (NSAIDs). including naproxen, effectively reverse incision-induced decreases in the MWT against mechanical hyperalgesia [20]. The plantar incision produced a marked mechanical hyperalgesia in the incised paw (paw withdrawal threshold diminished from $56.22 \pm 11.35 \mathrm{~g}$ at baseline to $1.17 \pm 1.29 \mathrm{~g}$ $24 \mathrm{~h}$ after plantar incision; $p<0.001)$. Administration of $H$. procumbens extracts $(300 \mathrm{mg} / \mathrm{kg}$, p.o.) significantly attenuated hyperalgesia in response to von Frey stimulation of the injured hind paw as 
evidenced by an increased mechanical withdrawal threshold (MWT) values as compared to control rats $(4.00 \pm 1.78 \mathrm{~g} v s .1 .17 \pm 1.29 \mathrm{~g}, p<0.05) 24 \mathrm{~h}$ after incision surgery. The group treated with 300 $\mathrm{mg} / \mathrm{kg}$ of H.procumbens extracts showed similar results as the group treated with $30 \mathrm{mg} / \mathrm{kg}$ of naproxen (Figure 1).

Figure 1. Effect of $H$. procumbens extracts on mechanical hyperalgesia induced by plantar incision in rats. Baseline assessment of animals, before surgery (day 0), showed no significant variation between groups. $6 \mathrm{~h}$ or $24 \mathrm{~h}$ after surgery, rats treated with H. procumbens extracts significantly attenuated hyperalgesia in response to von Frey stimulation of injured hind paw. Data are mean \pm SEM ( $=6$ per group). ${ }^{*} p<0.05$, significant difference from the control group.

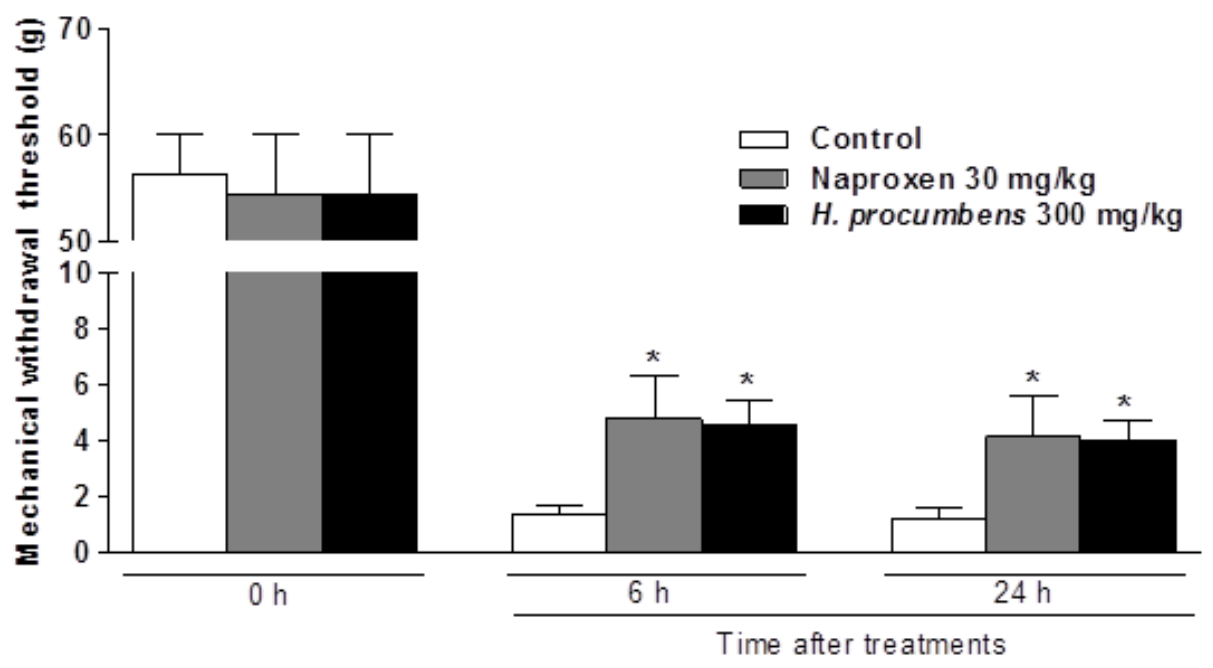

\subsection{Effects of H. Procumbens Extracts on Ultrasonic Vocalizations (USVs) Induced by Plantar Incision}

The anti-nociceptive activity of $H$. procumbens extracts was also determined by the pain-induced ultrasonic vocalizations (USVs) using ultrasonic microphones. Adult rats produce two distinct types of USVs that appear to reflect the caller's emotional state: either positive (a high-pitched and short $~ 50$ $\mathrm{kHz}$ USVs) or negative state (a low-pitched and longer $\sim 27 \mathrm{kHz}$ USV) [21]. In particular, 22-27 kHz USVs have been suggested as being a measure of affective shifts in rats [22] and have been used in a variety of unconditioned models such as pain, anxiety and stress-related models [23,24]. Pain-induced USVs have been examined by ultrasonic microphones, because vocalization is not only an objective but also a quantifiable value in the rats. After $6 \mathrm{~h}$ or $24 \mathrm{~h}$ after plantar incision, the control group emitted 22-27 kHz USVs calls, in pain-related behaviors [25]. The group treated with $30 \mathrm{mg} / \mathrm{kg}$ of naproxen showed significantly reduced 22-27 kHz USV calls compared with the control group, showing its antinociceptive effects in rats. $H$. procumbens extracts also reduced $22-27 \mathrm{kHz}$ USVs; a significant reduction was observed after the administration of $H$. procumbens extracts at $300 \mathrm{mg} / \mathrm{kg}(73.5 \%$, $p<0.01 v s$. control) (Figure 2). According to our findings, compared to the experimental group treated with naproxen, the $H$. procumbens extrcts might have anti-nociceptive effects on plantar incision postoperative pain in rats. 
Figure 2. Effect of $H$. procumbens extracts on USVs induced by plantar incision in rats. The sonograms of USVs in (A) control, (B) naproxen- and (C) H. procumbens extract- treated rats. (D) A significant difference in $22-27 \mathrm{kHz}$ USVs was observed between the $H$. procumbens extracts $(300 \mathrm{mg} / \mathrm{kg})$ treated group and the control group. Data are mean $\pm \operatorname{SEM}\left(\mathrm{n}=6\right.$ per group). ${ }^{*} p<0.05$, significant difference from the control group.
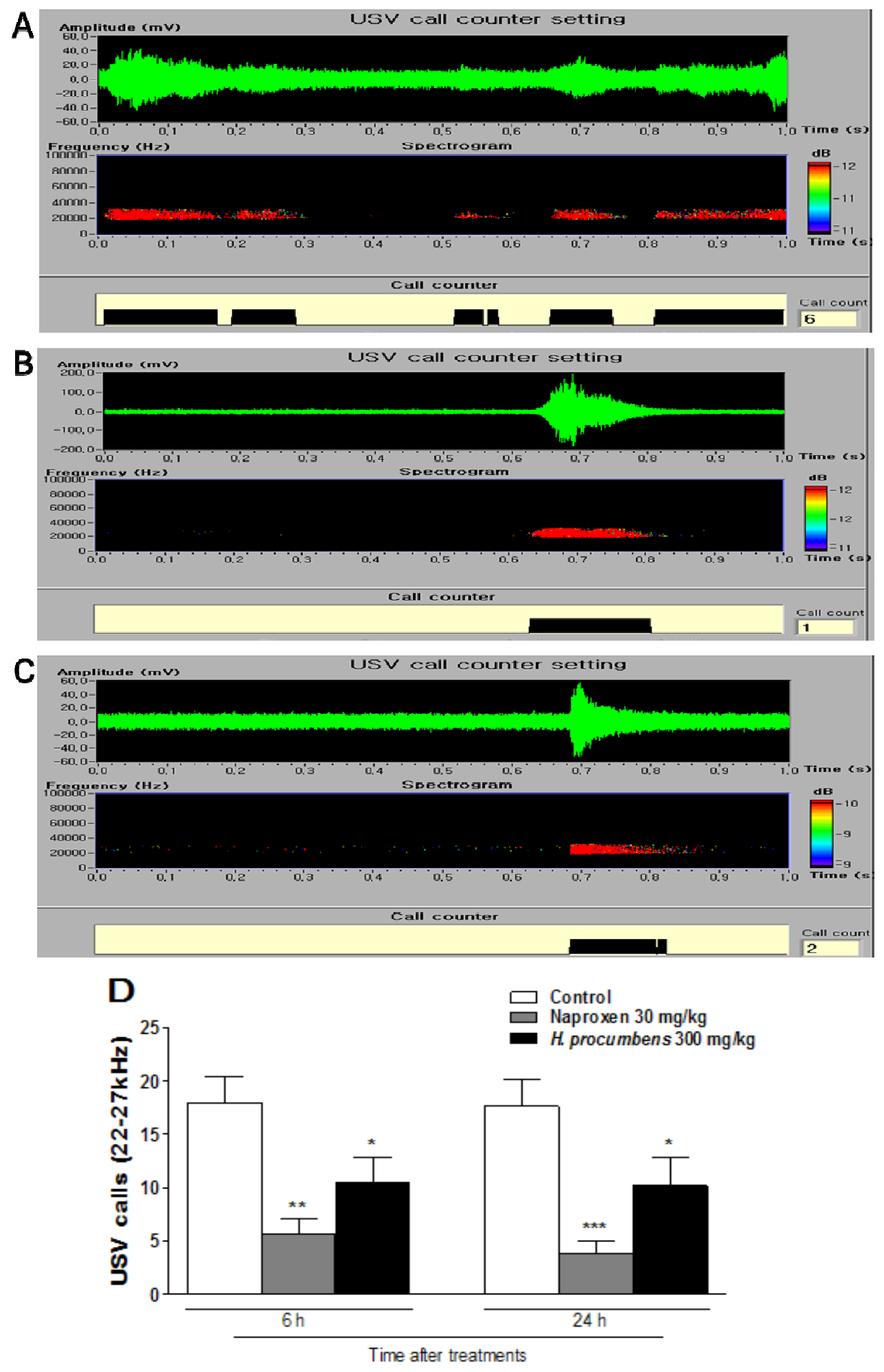


\subsection{Effects of H. Procumbens Extracts on Mechanical Allodynia Induced by Spared Nerve Injury (SNI)}

In this study we evaluated the potential efficacy of $H$. procumbens extracts in a rat model of the spared nerve injury (SNI) with regard to neuropathic pain. SNI mimics the symptoms of chronic nerve compression in humans [26]. At baseline (day 0), there are no significant changes between the H. procumbens extract (300 mg/kg)-treated group and SNI-control group (Figure 3). Animals began to show hypersensitivity responses to von Frey stimulation 3 days of after operation during the experiments. The frequency of withdrawals in the SNI-control group increased significantly reached from $56.22 \pm 3.77 \mathrm{~g}$ day 0 to $0.14 \pm 0.05 \mathrm{~g}$ on day 21 after SNI-operation. Administration of $H$. procumbens extracts $(300 \mathrm{mg} / \mathrm{kg})$ significantly attenuated allodynia in response to von Frey stimulation of the hind paw, as evidenced by increased MWT values as compared to SNI-control rats from 3 to 21 day after treatment, with an inhibition of $83.6 \%(p<0.001)$.

Figure 3. Anti-allodynic effects of $H$. procumbens extracts treatment in SNI rat model of neuropathic pain. Administration of $H$. procumbens extracts $(300 \mathrm{mg} / \mathrm{kg}$, p.o $)$ significantly attenuated allodynia in response to von Frey stimulation of hind paw from 3 to 21 day after treatment. Data are mean $\pm \operatorname{SEM}(\mathrm{n}=6$ per group). *** $p<0.001, * * p<0.01$, and $* p<0.05$ significant difference from control group.

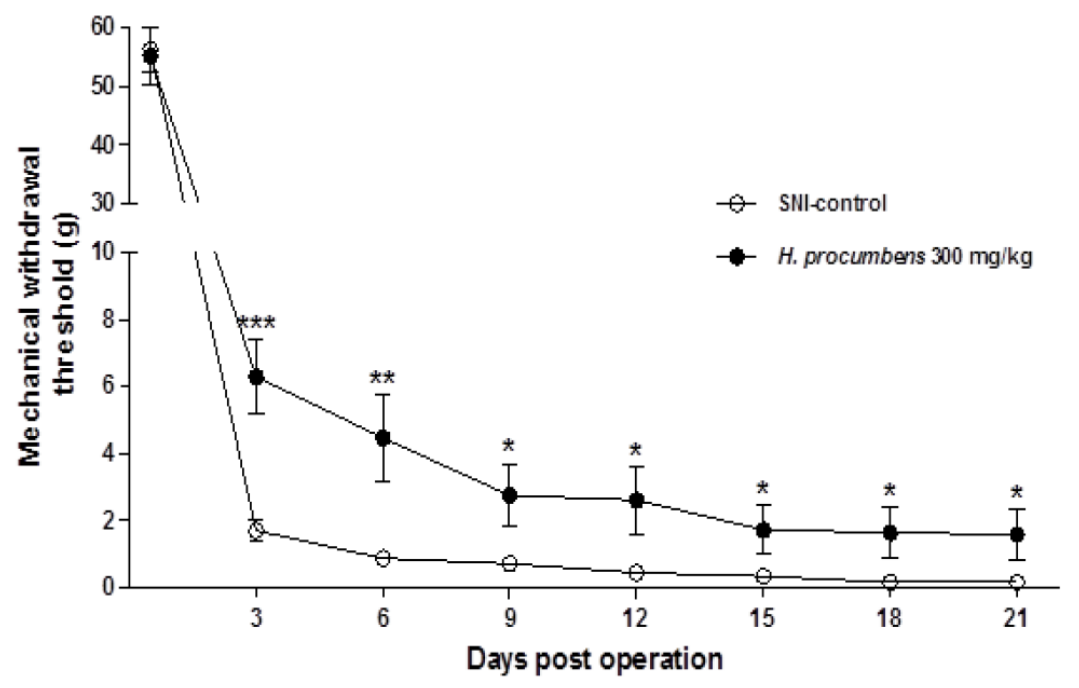

Wells et al. demonstrated that TNF (tumor necrosis factor) is increased in plasma after nerve crushing [27], and endoneurial injection of TNF- $\alpha$ can produce neuropathic pain behavior [28]. Also, Cyclooxygenase-2 (COX-2) is one of the important rate-limiting enzymes in the synthesis of prostaglandin (PG), a pain inducing substance, and is also the target of many non-steroidal analgesics. A study has showed significantly increased COX-2 positive cells on the first day and from day 7 to 14 following nerve injury, which is associated the formation of neuropathic pain [29]. It has been reported that both an ethanolic extract and an aqueous extract of $H$. procumbens prevented TNF- $\alpha$ synthesis; the latter, however, having a greater inhibitory effect on COX-2 pathway products [30,31]. Taken together, it might be hypothesized that $H$. procumbens extracts attenuated the behavioral symptoms of neuropathic pain in rats. 


\section{Experimental}

\subsection{Preparation of H. Procumbens Extracts}

H. procumbens $(300 \mathrm{~g})$ was extracted with $70 \%$ ethanol $(3 \mathrm{~L})$ for $4 \mathrm{~h}$ at $80{ }^{\circ} \mathrm{C}$ in a reflux apparatus. The process was repeated twice, and the extracts were filtered through membrane filters $(0.45 \mu \mathrm{m}$; Millipore, Billerica, MA, USA). The samples were lyophilized to yield a dark yellow powder. The yield of $H$. procumbens extracts was $41.6 \%$.

\subsection{Animals and Treatments}

Male Sprague-Dawley (SD) rats (160-200 g) were purchased from Samtako (Gyeonggi-do, Korea). Animals were housed at two rats per cage in an air-conditioned room at $23 \pm 1{ }^{\circ} \mathrm{C}, 55 \%-60 \%$ relative humidity, and a $12 \mathrm{~h}$ light/dark cycle (07:00 lights on, 19:00 lights off), and were given a regular laboratory rodent diet. After acclimatization for 1 week, 8-week-old male SD rats were anesthetized with $2 \%$ of isoflurane and pain-related surgeries were performed. After plantar incision operation, rats were divided into three following treatment groups: (1) control + vehicle, (2) control + naproxen (30 mg/kg, i.p.), and (3) control + H. procumbens extracts $(300 \mathrm{mg} / \mathrm{kg}$, p.o.). H. procumbens extracts and naproxen were dissolved in distilled water for oral administration at the desired doses in a volume of $5 \mathrm{~mL} / \mathrm{kg}$. The dosage of $H$. procumbens extracts was selected in consideration of usual human dosages (1.5-9 g/60 kg-weighted human, extracts dosages) commonly used in herbal medicine practice [12,32]. H. procumbens extracts at a dosage of $300 \mathrm{mg} / \mathrm{kg}$ in rats corresponds to $1.8 \mathrm{~g}$ $H$. procumbens extracts $/ 60 \mathrm{~kg}$-weight human subject, corresponding to $H$. procumbens extracted from approximately $4.3 \mathrm{~g}$ of the $H$. procumbens raw material. The sample treated groups were oral administrated $H$. procumbens extracts or naproxen after the plantar incision operations. After spared nerve injury (SNI) operation, rats were divided into two following treatment groups: (1) SNI-control + vehicle, and (2) SNI-control $+H$. procumbens extracts $(300 \mathrm{mg} / \mathrm{kg})$. H. procumbens extracts was given by p.o. route, immediately following surgery, once a day, which continued for 21 consecutive days. All animal experiments were carried out according to the guidelines of the Korea Food Research Institutional Animal Care and Use Committee.

\subsection{Plantar Incision of Postoperative Pain Rat Model}

Surgery was performed as previously described [16], with minor modifications. Briefly, rats were anaesthetized with $2 \%$ isofluorane and, a $1 \mathrm{~cm}$ longitudinal incision was made with scalpel, through skin and fascia of the plantar aspect of the paw, starting $0.5 \mathrm{~cm}$ from the proximal edge of the heel and extending toward the toes. The plantar muscle was raised and incised longitudinally. Following haemostasis with gentle pressure, the skin was opposed with two single interrupted sutures using polyamide monofilaments. The animals were allowed to recover in their home cages.

\subsection{Spared Nerve Injury (SNI) of Neuropathic Pain Rat Model}

The surgical procedure was performed as described previously with some modifications [33]. The SNI procedure comprised an axotomy and ligation of the tibial and common peroneal nerves leaving 
the sural nerve intact. The common peroneal and the tibial nerves were tightly ligated with 5.0 silk and sectioned distal to the ligation, removing $2 \pm 4 \mathrm{~mm}$ of the distal nerve stump. Great care was taken to avoid any contact with or stretching of the intact sural nerve. The skin was opposed with two single interrupted sutures using polyamide monofilaments.

\subsection{Mechanical Withdrawal Threshold (MWT) Analysis}

Animals were placed on an elevated wire grid and the plantar surface of the paw stimulated with a series of ascending force von Frey monofilaments (Stoelting, Chicago, IL, USA). The threshold was taken as the lowest force that evoked a brisk withdrawal response to one of three repetitive stimuli. To determine the time course of hyperalgesia and allodynia, a baseline measurement was made prior to surgery, and then again at 6 and 24 h post-surgery for hyperalgesia, 3, 6, 9, 12, 15, 18, and 21 days post-surgery for allodynia.

\subsection{Ultrasonic Vocalization (USVS) Analysis}

After induction of the plantar incision of postoperative pain, the category of $22-27 \mathrm{kHz} \mathrm{USVs}$ emitted by adult rats was monitored and scored for 10 min using Sonotrack ultrasonic microphones (Metris B.V., KA Hoofddorp, The Netherlands) placed at a distance of $25-30 \mathrm{~cm}$ from the heads of the animals. The rats emitted 'calls' that were counted using the Sonotrack 2.2.1 software.

\subsection{Statistical Analysis}

Data analyses were performed using one-way analysis of variance (ANOVA), followed by Tukey's post hoc test, using Prism 5 (GraphPad Software, Inc., San Diego, CA, USA) for multigroup comparisons. All data are presented as the mean \pm standard error (SEM). Significance was set at $p<0.05$.

\section{Conclusions}

In conclusion, the analgesic effect of $H$. procumbens extracts led to a reduction in the number of ultrasonic distress vocalizations caused by plantar incision postoperative pain in rats, and to a decrease in allodynia in response to von Frey stimulation of the hind paw evidenced by a decreased mechanical withdrawal threshold (MWT) in the spared nerve injury (SNI) neuropathic pain rat model. These results suggest that $H$. procumbens extracts could be useful on the treatment of postoperative and neuropathic pain, but more pharmacological and toxicological investigations are needed for finding the exact mechanism of action and for safety evaluation.

\section{Acknowledgments}

This study was supported by Grant from the Korea Food Research Institute.

\section{Conflicts of Interest}

The authors declare no conflict of interest. 


\section{References}

1. Stewart, W.F.; Ricci, J.A.; Chee, E.; Hahn, S.R.; Morganstein, D. Cost of lost productive work time among us workers with depression. JAMA 2003, 289, 3135-3144.

2. Koltzenburg, M.; Scadding, J. Neuropathic pain. Curr. Opin. Neurol. 2001, 14, 641-647.

3. Woolf, C.J.; Doubell, T.P. The pathophysiology of chronic pain--increased sensitivity to low threshold a beta-fibre inputs. Curr. Opin. Neurol. 1994, 4, 525-534.

4. Dickenson, A.H. Recent advances in the physiology and pharmacology of pain: Plasticity and its implications for clinical analgesia. J. Psychopharmacol. 1991, 5, 342-351.

5. Gilron, I.; Coderre, T.J. Emerging drugs in neuropathic pain. Expert Opin. Emerg. Drugs 2007, 12, 113-126.

6. Rao, P.; Knaus, E.E. Evolution of nonsteroidal anti-inflammatory drugs (nsaids): Cyclooxygenase (cox) inhibition and beyond. J. Pharm. Pharm. Sci. 2008, 11, 81s-110s.

7. Furlan, A.D.; Sandoval, J.A.; Mailis-Gagnon, A.; Tunks, E. Opioids for chronic noncancer pain: A meta-analysis of effectiveness and side effects. Can. Med. Assn. J. 2006, 174, 1589-1594.

8. Da Silva, K.A.; Manjavachi, M.N.; Paszcuk, A.F.; Pivatto, M.; Viegas, C., Jr.; Bolzani, V.S.; Calixto, J.B. Plant derived alkaloid (-)-cassine induces anti-inflammatory and anti-hyperalgesics effects in both acute and chronic inflammatory and neuropathic pain models. Neuropharmacology 2012, 62, 967-977.

9. Liu, M.; Zhou, L.; Chen, Z.; Hu, C. Analgesic effect of iridoid glycosides from paederia scandens (lour.) merrill (rubiaceae) on spared nerve injury rat model of neuropathic pain. Pharmacol. Biochem. Behav. 2012, 102, 465-470.

10. Yimam, M.; Brownell, L.; Hodges, M.; Jia, Q. Analgesic effects of a standardized bioflavonoid composition from scutellaria baicalensis and acacia catechu. J. Diet. Suppl. 2012, 9, 155-165.

11. Georgiev, M.I.; Alipieva, K.; Orhan, I.E. Cholinesterases inhibitory and antioxidant activities of harpagophytum procumbens from in vitro systems. Phytother. Res. 2012, 26, 313-316.

12. Brendler, T.; Gruenwald, J.; Ulbricht, C.; Basch, E.; Devil's claw (harpagophytum procumbens dc): An evidence-based systematic review by the natural standard research collaboration. J. Herbal Pharmacother. 2006, 6, 89-126.

13. Lanhers, M.C.; Fleurentin, J.; Mortier, F.; Vinche, A.; Younos, C. Anti-inflammatory and analgesic effects of an aqueous extract of harpagophytum procumbens. Planta Med. 1992, 58, 117-123.

14. Baghdikian, B.; Lanhers, M.C.; Fleurentin, J.; Ollivier, E.; Maillard, C.; Balansard, G.; Mortier, F. An analytical study, anti-inflammatory and analgesic effects of harpagophytum procumbens and harpagophytum zeyheri. Planta Med. 1997, 63, 171-176.

15. Andersen, M.L.; Santos, E.H.; Seabra Mde, L.; da Silva, A.A.; Tufik, S. Evaluation of acute and chronic treatments with harpagophytum procumbens on freund's adjuvant-induced arthritis in rats. J. Ethnopharmacol. 2004, 91, 325-330.

16. Brennan, T.J.; Vandermeulen, E.P.; Gebhart, G.F. Characterization of a rat model of incisional pain. Pain 1996, 64, 493-501.

17. Bourquin, A.F.; Suveges, M.; Pertin, M.; Gilliard, N.; Sardy, S.; Davison, A.C.; Spahn, D.R.; Decosterd, I. Assessment and analysis of mechanical allodynia-like behavior induced by spared nerve injury (sni) in the mouse. Pain 2006, 122, e11-e14. 
18. Swiergiel, A.H.; Zhou, Y.; Dunn, A.J. Effects of chronic footshock, restraint and corticotropin-releasing factor on freezing, ultrasonic vocalization and forced swim behavior in rats. Behav. Brain Res. 2007, 183, 178-187.

19. Sances, G.; Martignoni, E.; Fioroni, L.; Blandini, F.; Facchinetti, F.; Nappi, G. Naproxen sodium in menstrual migraine prophylaxis: A double-blind placebo controlled study. Headache 1990, 30, 705-709.

20. Whiteside, G.T.; Harrison, J.; Boulet, J.; Mark, L.; Pearson, M.; Gottshall, S.; Walker, K. Pharmacological characterisation of a rat model of incisional pain. Brit. J. Pharmacol. 2004, 141, 85-91.

21. Litvin, Y.; Blanchard, D.C.; Blanchard, R.J. Rat $22 \mathrm{khz}$ ultrasonic vocalizations as alarm cries. Behav. Brain Res. 2007, 182, 166-172.

22. Knutson, B.; Burgdorf, J.; Panksepp, J. Ultrasonic vocalizations as indices of affective states in rats. Psychol. Bull. 2002, 128, 961-977.

23. Brudzynski, S.M.; Chiu, E.M. Behavioural responses of laboratory rats to playback of $22 \mathrm{khz}$ ultrasonic calls. Physiol. Behav. 1995, 57, 1039-1044.

24. Miczek, K.A.; Weerts, E.M.; Vivian, J.A.; Barros, H.M. Aggression, anxiety and vocalizations in animals: Gabaa and 5-ht anxiolytics. Psychopharmacology 1995, 121, 38-56.

25. Portfors, C.V. Types and functions of ultrasonic vocalizations in laboratory rats and mice. J. Am. Assoc. Lab. Anim. Sci. 2007, 46, 28-34.

26. Richner, M.; Bjerrum, O.J.; Nykjaer, A.; Vaegter, C.B. The spared nerve injury (sni) model of induced mechanical allodynia in mice. J. Vis. Exp. 2011, doi:10.3791/3092.

27. Wells, M.R.; Racis, S.P., Jr.; Vaidya, U. Changes in plasma cytokines associated with peripheral nerve injury. J. Neuroimmunol. 1992, 39, 261-268.

28. Wagner, R.; Myers, R.R. Endoneurial injection of tnf-alpha produces neuropathic pain behaviors. Neuroreport 1996, 7, 2897-2901.

29. Takahashi, M.; Kawaguchi, M.; Shimada, K.; Konishi, N.; Furuya, H.; Nakashima, T. Cyclooxygenase-2 expression in schwann cells and macrophages in the sciatic nerve after single spinal nerve injury in rats. Neurosci. Lett. 2004, 363, 203-206.

30. Fiebich, B.L.; Heinrich, M.; Hiller, K.O.; Kammerer, N. Inhibition of tnf-alpha synthesis in lps-stimulated primary human monocytes by harpagophytum extract steihap 69. Phytomedicine 2001, 8, 28-30.

31. Kundu, J.K.; Mossanda, K.S.; Na, H.K.; Surh, Y.J. Inhibitory effects of the extracts of sutherlandia frutescens (1.) r. Br. And harpagophytum procumbens dc. On phorbol ester-induced cox-2 expression in mouse skin: Ap-1 and creb as potential upstream targets. Cancer Lett. 2005, 218, 21-31.

32. Erdos, A.; Fontaine, R.; Friehe, H.; Durand, R.; Poppinghaus, T. Contribution to the pharmacology and toxicology of different extracts as well as the harpagosid from harpagophytum procumbens dc (author's transl). Planta Med. 1978, 34, 97-108.

33. Decosterd, I.; Woolf, C.J. Spared nerve injury: An animal model of persistent peripheral neuropathic pain. Pain 2000, 87, 149-158.

Sample Availability: Samples of the H. procumbens extracts are available from the authors.

(C) 2014 by the authors; licensee MDPI, Basel, Switzerland. This article is an open access article distributed under the terms and conditions of the Creative Commons Attribution license (http://creativecommons.org/licenses/by/3.0/). 